\title{
PROPER COVERS OF RESTRICTION SEMIGROUPS AND $W$-PRODUCTS
}

\author{
MÁRIA B. SZENDREI
}

\begin{abstract}
Each factor semigroup of a free restriction (ample) semigroup over a congruence contained in the least cancellative congruence is proved to be embeddable into a $W$-product of a semilattice by a monoid. Consequently, it is established that each restriction semigroup has a proper (ample) cover embeddable into such a $W$-product.
\end{abstract}

\section{INTRODUCTION}

A restriction semigroup is a semigroup equipped with two additional unary operations which satisfy certain identities. For example, each inverse semigroup induces a restriction semigroup. Therefore restriction semigroups are considered non-regular generalizations of inverse semigroups. One-sided versions of restriction semigroups, where only one additional unary operation is considered, have been studied from various points of view, and under different names, since the 1960's. Restriction semigroups were formerly called weakly $E$-ample semigroups, and an important subclass is that of ample semigroups. For a historical overview, and for detailed introduction in the basic properties of these structures, the reader is referred to [2] and [4].

From the universal algebraic point of view, a restriction semigroup is an algebra of type $(2,1,1)$. Among others, the defining identities imply that both unary operations assign an idempotent to any element, and the ranges of the two unary operations coincide. This common range is the set of projections. The class of restriction semigroups forms a variety, and so it has free objects on any non-empty set $X$. A transparent model for the free restriction semigroup on $X$ is given in [2] as a subsemigroup in the free inverse semigroup on $X$. Moreover, the free restriction semigroup on $X$ is proved to be embeddable in a semidirect-like product of a semilattice by the free monoid $X^{*}$ where $X^{*}$ acts on the semilattice both on the left and on the right.

In this paper we present a similar embedding theorem for a more general family of restriction semigroups, called sometimes quasi-free ([2]), where another semidirect-like product is used whose definition requires a single action. The family of restriction semigroups we consider consists of the factor semigroups of the free restriction semigroups over congruences contained in the least cancellative congruence. The construction called a $W$-product

\footnotetext{
Research supported by the Hungarian National Foundation for Scientific Research grant no. SAB81142.

Mathematical Subject Classification (2010): 20M10, $20 \mathrm{M} 05$.

$K e y$ words: Restriction semigroup, weakly $E$-ample semigroup, proper cover, $W$-product, free, quasi-free.
} 
which is involved in our main result was introduced in [1], and played a central role also in [3]. As a consequence, we deduce that each restriction semigroup can be obtained as a (projection separating) homomorphic image of a restriction subsemigroup in a $W$-product of a semilattice by a monoid. Since a $W$-product of a semilattice by a group is just the usual semidirect product of them, this generalizes a well-known fact of the structure theory of inverse semigroups. Another construction of each restriction semigroup from a $W$-product of a semilattice by a monoid is proved in [6] where the operators of 'taking a restriction subsemigroup' and 'taking a homomorphic image' are applied in the reverse order.

In Section 3, we present a model of the free restriction semigroup on $X$ as a restriction subsemigroup in a $W$-product of a semilattice by the free monoid $X^{*}$. In Section 4, we characterize the congruences of a free restriction semigroup contained in the least cancellative congruence, and apply this to show that any factor semigroup over such a congruence is embeddable in a $W$-product of a semilattice by a monoid. Finally, we deduce the result mentioned above that constructs every restriction semigroup from such a $W$-product.

\section{Preliminaries}

In this section the basic notions and facts on restriction semigroups are provided. For more details, the reader is referred to [4]. Furthermore, we recall the definition of the $W$-product construction and its most important properties. Finally, we present the model of the free restriction semigroup on a set $X$ given in [2].

A left restriction semigroup is defined to be an algebra of type $(2,1)$, more precisely, an algebra $S=\left(S ; \cdot,{ }^{+}\right)$where $(S ; \cdot)$ is a semigroup and ${ }^{+}$is a unary operation such that the following identities are satisfied:

$$
x^{+} x=x, \quad x^{+} y^{+}=y^{+} x^{+}, \quad\left(x^{+} y\right)^{+}=x^{+} y^{+}, \quad x y^{+}=(x y)^{+} x .
$$

A right restriction semigroup is defined dually, that is, it is an algebra $S=$ $\left(S ; \cdot,{ }^{*}\right)$ satisfying the duals of the identities $(2.1)$. If $S=\left(S ; \cdot,{ }^{+},{ }^{*}\right)$ is an algebra of type $(2,1,1)$ where $S=\left(S ; \cdot,{ }^{+}\right)$is a left restriction semigroup, $S=\left(S ; \cdot,{ }^{*}\right)$ is a right restriction semigroup and the identities

$$
\left(x^{+}\right)^{*}=x^{+}, \quad\left(x^{*}\right)^{+}=x^{*}
$$

hold then it is called a restriction semigroup. By definition, it is obvious that the class of restriction semigroups forms a variety of algebras of type $(2,1,1)$. The notions of a subalgebra, homomorphism, congruence and factor algebra are understood in this type. In order to emphasize this, we use the expressions $(2,1,1)$-subsemigroup, $(2,1,1)$-morphism, $(2,1,1)$-congruence and $(2,1,1)$-factor semigroup, respectively.

If a restriction semigroup $S$ has an identity element 1 with respect to the multiplication then it is straightforward to see by (2.1) that

$$
1^{+}=1^{*}=1 \text {. }
$$

Such a restriction semigroup is called a restriction monoid. A restriction monoid can be considered an algebra of type $(2,1,1,0)$ where the nullary operation picks out the identity element. 
The class of restriction semigroups is fairly big. For example, each inverse semigroup $S_{\mathrm{inv}}=\left(S ; \cdot,{ }^{-1}\right)$ determines a restriction semigroup $S=\left(S ; \cdot,^{+},{ }^{*}\right)$ where the unary operations are defined in the following manner:

$$
a^{+}=a a^{-1} \quad \text { and } \quad a^{*}=a^{-1} a \text { for every } a \in S .
$$

By the Wagner-Preston theorem, such a restriction semigroup is, up to $(2,1,1)$-isomorphism, a $(2,1,1)$-subsemigroup of $\left(I(X) ; \cdot,,^{+},{ }^{*}\right)$ for some set $X$, where $I(X)$ is the set of all partial bijections on $X$, and

$$
\alpha^{+}=1_{\operatorname{dom} \alpha} \quad \text { and } \quad \alpha^{*}=1_{\operatorname{im} \alpha} \quad \text { for every } \alpha \in I(X) .
$$

On the other hand, each monoid $M$ becomes a restriction semigroup by defining $a^{+}=a^{*}=1$ for any $a \in M$. It is easy to see that these restriction semigroups are just those with both unary operations being constant. Such a restriction semigroup will be called unary trivial, and, since it is necessarily a monoid, we also call it a unary trivial restriction monoid. In the literature, unary trivial restriction semigroups (monoids) are sometimes called reduced restriction semigroups (monoids). Notice that the congruences, homomorphisms, etc. of monoids and the $(2,1,1)$-congruences, $(2,1,1)$-morphisms of the unary trivial monoids corresponding to them are the same. Therefore we often consider unary trivial semigroups just as monoids, and vice versa.

Let $S$ be any restriction semigroup. By (2.2), we have $\left\{x^{+}: x \in S\right\}=$ $\left\{x^{*}: x \in S\right\}$. This set is called the set of projecions of $S$, and is denoted by $P_{S}$. It is easy to see that $P_{S}$ is a $(2,1,1)$-subsemilattice in $S$, and both unary operations are identical on it. In particular $P_{S}$ consists of idempotent elements of $S$ only. Notice that a restriction semigroup $S$ is unary trivial if and only if $P_{S}$ is a singleton, and, if this is the case then the unique element of $P_{S}$ is the identity element of $S$. If $S, T$ are restriction semigroups, and $\phi: S \rightarrow T$ is a $(2,1,1)$-morphism then $\phi$ is said to be projection separating if $e \phi=f \phi$ implies $e=f$ for every $e, f \in P_{S}$.

We consider a relation on $S$, denoted by $\sigma$, which is defined, for any $a, b \in S$, by the rule

$$
a \sigma b \text { if and only if } e a=e b \text { for some } e \in P_{S} .
$$

Notice that if there exists $e \in P_{S}$ with $e a=e b$ then there exists also $f \in P_{S}$ with $a f=b f$, and conversely. Therefore the relation defined dually to $\sigma$ coincides with $\sigma$. The relation $\sigma$ is the least congruence on $S=(S ; \cdot)$ where $P_{S}$ is in a congruence class, which we denote by $P_{S} \sigma$. Consequently, $\sigma$ is the least $(2,1,1)$-congruence $\rho$ on $S=\left(S ; \cdot,{ }^{+},{ }^{*}\right)$ such that the $(2,1,1)$-factor semigroup $S / \rho$ is unary trivial. Therefore we call $\sigma$ the least unary trivial $(2,1,1)$-congruence on $S$. The unary trivial restriction monoid $S / \sigma$ is often considered just as a monoid $S / \sigma=\left(S / \sigma ; \cdot, P_{S} \sigma\right)$ with identity element $P_{S} \sigma$.

A left ample semigroup is defined to be a left restriction semigroup $S$ where, for any $a, b \in S$, we have

$$
a^{+}=b^{+} \quad \text { if and only if } \quad x a=y a \Longleftrightarrow x b=y b \text { for all } x, y \in S^{1} .
$$

A right ample semigroup is defined dually, and by an ample semigroup we mean a restriction semigroup that is both left and right ample. On a (left) ample semigroup, the relation $\sigma$ is the least (right) cancellative congruence. Ample semigroups form a sub-quasivariety in the variety of restriction semigroups. 
A restriction semigroup $S$ is said to be proper if the following condition and its dual are fulfilled:

$$
a^{+}=b^{+} \text {and } a \sigma b \quad \text { imply } \quad a=b \quad \text { for every } a, b \in S .
$$

If $S, T$ are restriction semigroups and $T$ is proper then $T$ is said to be a proper cover of $S$ if there exists a projection separating and surjective $(2,1,1)$-morphism from $T$ onto $S$.

It is worth mentioning that if a restriction semigroup $S$ is obtained from an inverse semigroup $S_{\mathrm{inv}}=\left(S ; \cdot,{ }^{-1}\right)$ as above then $\sigma$ is the least group congruence on $S_{\mathrm{inv}}$, and $S$ is proper if and only if $S_{\mathrm{inv}}$ is $E$-unitary.

The construction of $W(T, Y)$ with $Y$ being a semilattice and $T$ a right cancellative monoid was introduced in [1] as a construction of a left ample semigroup. In [3], it was generalized for any unipotent monoid $T$, and it was noticed that there is a natural unary operation * on $W(T, Y)$, so that it becomes a so-called weakly ample semigroup. A weakly ample semigroup is a restriction semigroup in which every idempotent is a projection. The arguments in [3] are easily seen to apply for any monoid $T$, and $W(T, Y)$ becomes a restriction semigroup (cf. [6]).

Let $T$ be a monoid and $Y$ a semilattice. We say that $T$ acts on $Y$ on the right [left] if a monoid homomorphism is given from $T$ into the endomorphism monoid End $Y$ of $Y$ [into the dual End ${ }^{d} Y$ of the endomorphism monoid of $Y]$. For brevity, $a^{t}\left[{ }^{t} a\right]$ is used to denote the image of the element $a \in Y$ under the endomorphism assigned to the element $t \in T$. This is equivalent to requiring that the equalities

$$
(a b)^{t}=a^{t} b^{t}, \quad\left(a^{t}\right)^{u}=a^{t u}, \quad a^{1}=a
$$

are valid for every $a, b \in Y$ and $t, u \in T$. Suppose that $T$ acts on the right on $Y$ by injective endomorphisms such that the range of each endomorphism corresponding to an element of $T$ forms an order ideal in $Y$. Equivalently, suppose that, additionally to (2.4), we have

$$
a^{t}=b^{t} \quad \text { implies } \quad a=b,
$$

and

$$
a \leq b^{t} \quad \text { implies } \quad a=c^{t} \text { for some } c \in Y
$$

for every $a, b \in Y$ and $t \in T$. Consider the set

$$
W(T, Y)=\left\{\left(t, a^{t}\right) \in T \times Y: a \in Y, t \in T\right\},
$$

and define a multiplication and two unary operations on it by the following rules: for any $\left(t, a^{t}\right),\left(u, b^{u}\right) \in W(T, Y)$, put

$$
\begin{aligned}
\left(t, a^{t}\right)\left(u, b^{u}\right) & =\left(t u, a^{t u} \cdot b^{u}\right), \\
\left(t, a^{t}\right)^{+} & =(1, a), \\
\left(t, a^{t}\right)^{*} & =\left(1, a^{t}\right) .
\end{aligned}
$$

It is straightforward to see that $W(T, Y)$ is a subsemigroup in the reverse semidirect product $T \ltimes Y$. We call $W(T, Y)$ a $W$-product of $Y$ by $T$. This construction has the following basic properties.

Result 2.1. Let $Y$ be a semilattice and $T$ a monoid acting on $Y$ on the right, so that conditions (2.5),(2.6) are fulfilled. 
(1) $W(T, Y)=\left(W(T, Y) ; \cdot,{ }^{+},{ }^{*}\right)$ is a restriction semigroup, its set of projections is $P_{W(T, Y)}=\{(1, a): a \in Y\}$, which is isomorphic to $Y$.

(2) The first projection $\pi: W(T, Y) \rightarrow T$ is a surjective homomorphism whose kernel is $\sigma$. Consequently, $W(T, Y) / \sigma$ is isomorphic to $T$.

(3) $W(T, Y)$ is proper.

(4) $W(T, Y)$ is a monoid if and only if $Y$ has an identity.

Given a class $\mathcal{V}$ of algebras of type $(2,1,1)$, a non-empty set $X$, a member $F \in \mathcal{V}$ and a mapping $f: X \rightarrow F$, we say that $(F, f)$, or, briefly, $F$ is a free object in $\mathcal{V}$ on $X$ if it possesses the following universal property: for any $V \in \mathcal{V}$ and any mapping $v: X \rightarrow V$, there is a unique $(2,1,1)$-morphism $\phi: F \rightarrow V$ such that $f \phi=v$. One can prove that $F$ is, up to $(2,1,1)$ isomorphism, unique, provided it exists. It is well known that if $\mathcal{V}$ is a variety then there exists a free object in $\mathcal{V}$ on any non-empty set. In particular, this ensures that there exists a free restriction semigroup on any non-empty set, and, up to $(2,1,1)$-isomorphism, it is uniquely determined. A model of the free restriction semigroup on $X$ is given in [2] as a full subsemigroup in the free inverse semigroup on $X$ (cf. [5]). We recall it in the rest of this section.

For any set $X$, consider the free monoid $X^{*}$ and the free group $F \mathcal{G}(X)$ on $X$. The elements of $X^{*}$ are said to be words in $X$. The multiplication in $X^{*}$ is juxtaposition. The identity element of $X^{*}$ is the empty word which we denote by 1 .

The elements of $F \mathcal{G}(X)$ are supposed to be the reduced words in $X \cup X^{-1}$. For any word $w \in\left(X \cup X^{-1}\right)^{*}$, the reduced form of $w$ is denoted by $\operatorname{red}(w)$. Thus the product of any elements $u, v$ in $F \mathcal{G}(X)$ is $\operatorname{red}(u v)$. Obviously, $X^{*}$ is a subsemigroup in $F \mathcal{G}(X)$ but $F \mathcal{G}(X)$ is not a sub(semi)group in $\left(X \cup X^{-1}\right)^{*}$.

Now we briefly recall a model for free inverse semigroups ([5]). The prefix order $\leq_{p}$ is a partial order defined on $F \mathcal{G}(X)$ by $u \leq_{p} v$ if $u$ is a prefix of $v$, that is, $v=u w(=\operatorname{red}(u w))$ for some $w \in F \mathcal{G}(X)$. If $A$ is a non-empty subset in $F \mathcal{G}(X)$ then $[A]^{\downarrow}$ denotes the order ideal of $\left(F \mathcal{G}(X) ; \leq_{p}\right)$ generated by $A$. In particular, $[u]^{\downarrow}$ is the set of all prefixes of the word $u(\in F \mathcal{G}(X))$ including 1 and $u$. Therefore each order ideal* but $[1]^{\downarrow}=\{1\}$ has at least two elements.

Denote by $\mathcal{Y}^{\bullet}$ the set of all finite order ideals of $\left(F \mathcal{G}(X) ; \leq_{p}\right)$, and put $\mathcal{Y}=\mathcal{Y}^{\bullet} \backslash\{\{1\}\}$. For any $v \in F \mathcal{G}(X)$ and any subset $S \subseteq F \mathcal{G}(X)$, define

$$
{ }^{v} S=\{\operatorname{red}(v s): s \in S\},
$$

and put $\mathcal{X}=F \mathcal{G}(X) \mathcal{Y}$. Consider the set

$$
P(F \mathcal{G}(X), \mathcal{X}, \mathcal{Y})=\{(A, u) \in \mathcal{Y} \times F \mathcal{G}(X): u \in A\},
$$

and equip it with the following multiplication:

$$
(A, u)(B, v)=\left(A \cup{ }^{u} B, u v\right) .
$$

Then $P(F \mathcal{G}(X), \mathcal{X}, \mathcal{Y})$ is an inverse semigroup, and, together with the injective mapping

$$
X \rightarrow P(F \mathcal{G}(X), \mathcal{X}, \mathcal{Y}), x \mapsto(\{1, x\}, x),
$$

*The empty set is not considered an order ideal. 
it is a free inverse semigroup on $X$. Observe that $P(F \mathcal{G}(X), \mathcal{X}, \mathcal{Y})$ is just the $P$-semigroup corresponding to the McAlister triple $(F \mathcal{G}(X), \mathcal{X}, \mathcal{Y})$, where $\mathcal{X}=(\mathcal{X} ; \supseteq)$, and $F \mathcal{G}(X)$ acts on it, by the rule $(2.8)$, on the left by order automorphisms.

It is well known that the Cayley graph $\Gamma F \mathcal{G}(X)$ of $F \mathcal{G}(X)$, as an $X$ generated group, is a tree, and $F \mathcal{G}(X)$ acts on $\Gamma F \mathcal{G}(X)$ on the left by translations, that is, by putting ${ }^{v}(u, x)=(v u, x)$ for any $v \in F \mathcal{G}(X)$ and any edge $(u, x)$. Recall that there is a natural bijection $\beta_{1}$ from $\mathcal{Y}$ onto the set of all finite connected subgraphs of $\Gamma F \mathcal{G}(X)$ containing the vertex 1 and at least one edge. Namely, $A \in \mathcal{Y}$ is the set of vertices of $A \beta_{1}$. Consequently, there is a natural bijection $\beta$ from $\mathcal{X}$ onto the set of all finite connected subgraphs of $\Gamma F \mathcal{G}(X)$ containing at least one edge where $S \in \mathcal{X}$ is the set of vertices of $S \beta$. In particular, we easily see that, for any $S \in \mathcal{X}$, we have $S \in \mathcal{Y}$ if and only if $1 \in S$. It is obvious that this bijection respects the left action of $F \mathcal{G}(X)$, that is, we have $\left({ }^{v} S\right) \beta={ }^{v}(S \beta)$ for every $v \in F \mathcal{G}(X)$ and $S \in \mathcal{X}$.

In some cases, this observation makes the constructions involving $\mathcal{Y}$ or $\mathcal{X}$ more transparent. For example, it easily follows from the fact that $\Gamma F \mathcal{G}(X)$ is a tree that, for any two finite connected subgraphs $\Delta$ and $\Theta$, there is a least finite connected subgraph containing $\Delta$ and $\Theta$. Denoting it $\Delta \vee \Theta$, it can be obtained as follows: if $u$ is a vertex in $\Delta, v$ is a vertex in $\Theta$, and $\Pi$ is the (finite connected) subgraph spanned by the unique reduced path in $\Gamma F \mathcal{G}(X)$ from $u$ to $v$, then $\Delta \vee \Theta=\Delta \cup \Pi \cup \Theta$. In particular, if $\Delta$ and $\Theta$ have a common vertex, then we have $\Delta \vee \Theta=\Delta \cup \Theta$. It is clear from this description of the operation $\vee$ that the left action of $F \mathcal{G}(X)$ on $\Gamma F \mathcal{G}(X)$ respects $\vee$, that is, ${ }^{v}(\Delta \vee \Theta)={ }^{v} \Delta \vee^{v} \Theta$ for any $v \in F \mathcal{G}(X)$ and any finite connected subgraphs $\Delta, \Theta$. These observations, translated into the language of order ideals of $\left(F \mathcal{G}(X) ; \leq_{p}\right)$, can be formulated as follows:

Result 2.2. Let $R, S$ be arbitrary elements in $(\mathcal{X} ; \supseteq)$.

(1) The elements $R$ and $S$ have a least upper bound $R \vee S$ in $(\mathcal{X} ; \supseteq)$, and we have

$$
R \vee S={ }^{r}\left(r^{-1} R \cup\left[r^{-1} s\right]^{\downarrow} \cup r^{-1} S\right)
$$

for every $r \in R$ and $s \in S$.

(2) In particular, $R \vee S=R \cup S$ provided $R, S \in \mathcal{X}$ such that $R \cap S \neq \emptyset$.

(3) We have ${ }^{v}(R \vee S)={ }^{v} R \vee{ }^{v} S$ for every $v \in F \mathcal{G}(X)$.

(4) Consequently, $\mathcal{X}=(\mathcal{X} ; \vee)$ is a semilattice acted upon on the left by $F \mathcal{G}(X)$ by automorphisms. Moreover, $\mathcal{Y}$ forms a subsemilattice in $i$, and the operations $\vee$ and $\cup$ coincide on $\mathcal{Y}$.

Notice that statement (2) allows us to replace "U" by "У" in (2.9).

Remark 2.3. In the argument above, we can replace the semilattice $\mathcal{Y}$ by the semilattice monoid $\mathcal{Y}^{\bullet}$ and $\mathcal{X}$ by $\mathcal{X}^{\bullet}=F \mathcal{G}(X) \mathcal{Y}^{\bullet}$. Then $P\left(F \mathcal{G}(X), \mathcal{X}^{\bullet}, \mathcal{Y}^{\bullet}\right)$ turns out to be the free inverse monoid on $X$. In particular, the relations mentioned remain valid for $\mathcal{X}^{\bullet}$ and for the set of all finite connected subgraphs of $\Gamma F \mathcal{G}(X)$ (with or without an edge), and Result 2.2 holds for $\left(\mathcal{X}^{\bullet} ; \supseteq\right)$ and $\left(\mathcal{X}^{\bullet} ; \vee\right)$. 
It was proved in [2] that the inverse image of the free monoid $X^{*}$, as a subsemigroup in $F \mathcal{G}(X)$, under the second projection of $P\left(F \mathcal{G}(X), \mathcal{X}^{\bullet}, \mathcal{Y}^{\bullet}\right)$ onto $F \mathcal{G}(X)$ is the free restriction monoid (see Remark 2.5 below). Here we present the semigroup analogue of this result.

For any non-empty set $X$, consider the set

$$
F \mathcal{R S}(X)=\left\{(A, u) \in \mathcal{Y} \times F \mathcal{G}(X): u \in X^{*} \cap A\right\},
$$

and define a multiplication by (2.9), and unary operations by

$$
(A, u)^{+}=(A, 1) \text { and }(A, u)^{*}=\left({ }^{u^{-1}} A, 1\right) .
$$

Result 2.4. (1) $F \mathcal{R S}(X)$ is a proper restriction semigroup, and it is ample.

(2) The second projection of $F \mathcal{R S}(X)$ is a $(2,1,1)$-morphism which induces the congruence $\sigma$.

(3) The set of projections of $F \mathcal{R} \mathcal{S}(X)$ is

$$
P_{F \mathcal{R}(X)}=\{(A, 1): A \in \mathcal{Y}\}
$$

which is isomorphic to $\mathcal{Y}$.

(4) The restriction semigroup $F \mathcal{R S}(X)$ together with the injective mapping

$$
X \rightarrow F \mathcal{R S}(X), x \mapsto(\{1, x\}, x)
$$

is a free restriction semigroup on $X$.

Remark 2.5. Define $F \mathcal{R} \mathcal{M}(X)$ by replacing $\mathcal{Y}$ by $\mathcal{Y}^{\bullet}$ and $\mathcal{X}$ by $\mathcal{X} \bullet$ in the definition of $F \mathcal{R S}(X)$. The analogue of Result 2.4 is valid and establishes that $F \mathcal{R} \mathcal{M}(X)$ is the free restriction monoid on $X$ which is ample.

By investigating the properties of a free restriction semigroup, the following important fact is deduced in [2] as an application of Result 2.4.

Result 2.6. Each restriction semigroup has a proper (ample) cover which is $(2,1,1)$-isomorphic to a $(2,1,1)$-factor semigroup of a free restriction semigroup over a $(2,1,1)$-congruence contained in the least unary trivial $(2,1,1)$ congruence (equivalently, in the least cancellative congruence).

A restriction semigroup being $(2,1,1)$-isomorphic to a $(2,1,1)$-factor semigroup of a free restriction semigroup over a $(2,1,1)$-congruence contained in the least unary trivial $(2,1,1)$-congruence (equivalently, in the least cancellative congruence) is termed in [2] quasi-free.

\section{Free Restriction Semigroups and $W$-products}

In this section we establish that each free restriction semigroup is $(2,1,1)$ embeddable in a $W$-product of a semilattice by a monoid.

Let $X$ be any non-empty set, and consider the free restriction semigroup $F \mathcal{R S}(X)$ on $X$. The left action of the group $F \mathcal{G}(X)$ on the semilattice $\mathcal{X}$ (see Result $2.2(3),(4)$ ) naturally defines a right action of $F \mathcal{G}(X)$ on $\mathcal{X}$ by the rule

$$
S^{u}={ }^{u^{-1}} S \quad(u \in F \mathcal{G}(X), S \in \mathcal{X}) .
$$

Its restriction to $X^{*}$ is clearly a right action of the monoid $X^{*}$ on the semilattice $\mathcal{X}$. Moreover, since $F \mathcal{G}(X)$ acts by automorphisms, the same holds for $X^{*}$. 
Define the following subset in $\mathcal{X}$ :

$$
\mathcal{Q}=\mathcal{Y}^{X^{*}}
$$

Obviously, we have

$$
\mathcal{Q}=\left\{Q \in \mathcal{X}: Q \cap\left(X^{*}\right)^{-1} \neq \emptyset\right\}
$$

where $T^{-1}$ is used to denote the subset $\left\{t^{-1}: t \in T\right\}$ in $F \mathcal{G}(X)$ for any $T \subseteq X^{*}$. It is straightforward that $\mathcal{Q}^{X^{*}} \subseteq \mathcal{Q}$, and, by the last equation, that $\mathcal{Q}$ is closed under forming supersets. Hence we obtain the following statement by Result 2.2(4).

Lemma 3.1. The subset $\mathcal{Q}$ forms a subsemilattice in $\mathcal{X}=(\mathcal{X} ; \vee)$, and the monoid $X^{*}$ acts on it on the right by injective endomorphisms.

Now we establish another important property of the right action of $X^{*}$ on $\mathcal{Q}$.

Lemma 3.2. For each $t \in X^{*}$, we have

$$
\mathcal{Q}^{t}=\left\{Q \in \mathcal{X}: Q \cap\left(X^{*} t\right)^{-1} \neq \emptyset\right\},
$$

and so it is a dual order ideal in $(\mathcal{Q} ; \supseteq)$.

Proof. In order to check the equality, first observe that if $P \in \mathcal{Q}$ then there exists $s \in X^{*}$ with $s^{-1} \in P$ whence we see that $s t \in X^{*}$ and $(s t)^{-1}=$ $t^{-1} s^{-1} \in{ }^{-1} P=P^{t}$. Conversely, if $S \in \mathcal{X}$ with $S \cap\left(X^{*} t\right)^{-1} \neq \emptyset$, say, $(s t)^{-1} \in S$ with $s \in X^{*}$ then $s^{-1}=t(s t)^{-1} \in{ }^{t} S$, and so $P={ }^{t} S \in \mathcal{Q}$ and $S={ }^{t^{-1}}\left({ }^{t} S\right)={ }^{t^{-1}} P=P^{t} \in \mathcal{Q}^{t}$. The second statement is immediately implied by this equality, since its right hand side is obviously closed under forming supersets.

Lemmas 3.1 and 3.2 imply that the $W$-product $W\left(X^{*}, \mathcal{Q}\right)$ is defined. Observe that

$$
W\left(X^{*}, \mathcal{Q}\right)=\left\{(t, Q) \in X^{*} \times \mathcal{Q}: Q \cap\left(X^{*} t\right)^{-1} \neq \emptyset\right\} .
$$

Now we formulate the main result of this section.

Theorem 3.3. The free restriction semigroup $F \mathcal{R S}(X)$ is $(2,1,1)$-embeddable in the $W$-product $W\left(X^{*}, \mathcal{Q}\right)$.

Proof. We show that the mapping

$$
\iota: F \mathcal{R S}(X) \rightarrow W\left(X^{*}, \mathcal{Q}\right),(A, t) \mapsto\left(t, A^{t}\right)
$$

is a $(2,1,1)$-embedding. First note that $\iota$ is well defined by $(3.1)$, and it is injective by Lemma 3.1. Now we check that $\iota$ is a $(2,1,1)$-morphism. We have

and

$$
((A, t)(B, u)) \iota=\left(A \cup^{t} B, t u\right) \iota=\left(t u,\left(A \cup{ }^{t} B\right)^{t u}\right)
$$

$$
(A, t) \iota(B, u) \iota=\left(t, A^{t}\right)\left(u, B^{u}\right)=\left(t u, A^{t u} \vee B^{u}\right)
$$

They are, indeed, equal since $t \in A$ by the definition of $F \mathcal{R S}(X)$, and so the following holds in $\mathcal{X}$ by the definition of the right action and by Result 2.2 :

$$
\begin{aligned}
\left(A \cup{ }^{t} B\right)^{t u} & ={ }^{(t u)^{-1}}\left(A \cup{ }^{t} B\right)=(t u)^{-1}\left(A \vee^{t} B\right) \\
& ={ }^{(t u)^{-1}} A{ }^{(t u)^{-1} t} B=(t u)^{-1} A \vee^{u^{-1}} B=A^{t u} \vee B^{u}
\end{aligned}
$$


Moreover, we have $\left((A, t)^{+}\right) \iota=(A, 1) \iota=(1, A)=\left(t, A^{t}\right)^{+}=((A, t) \iota)^{+}$and $\left((A, t)^{*}\right) \iota=\left(t^{-1} A, 1\right) \iota=\left(1,{ }^{-1} A\right)=\left(1, A^{t}\right)=\left(t, A^{t}\right)^{*}=((A, t) \iota)^{*}$.

An immediate consequence of this theorem is that $F \mathcal{R} \mathcal{S}(X) \iota$ is a $(2,1,1)$ subsemigroup in $W\left(X^{*}, \mathcal{Q}\right)$. This implies the following statement.

Consider the subset

$$
\begin{aligned}
F_{W} \mathcal{R S}(X) & =\left\{\left(t, A^{t}\right) \in W\left(X^{*}, \mathcal{Q}\right): 1, t \in A\right\} \\
& =\left\{\left(t, A^{t}\right) \in X^{*} \times \mathcal{Y}: A \in \mathcal{Y}\right\} \\
& =\left\{(t, B) \in X^{*} \times \mathcal{Y}: t^{-1} \in B\right\}
\end{aligned}
$$

in $W\left(X^{*}, \mathcal{Q}\right)$.

Corollary 3.4. The subset $F_{W} \mathcal{R S}(X)$ forms a $(2,1,1)$-subsemigroup in $W\left(X^{*}, \mathcal{Q}\right)$. Furthermore, $F_{W} \mathcal{R S}(X)$ together with the injective mapping

$$
X \rightarrow F_{W} \mathcal{R S}(X), x \mapsto\left(x,\left\{1, x^{-1}\right\}\right)
$$

is a free restriction semigroup on $X$.

Remark 3.5. (1) Defining $\mathcal{Q}^{\bullet}$ to be $\mathcal{Y}^{\bullet} X^{*}$, we obtain a subsemilattice in $\mathcal{X}^{\bullet}=\left(\mathcal{X}^{\bullet} ; \vee\right)$. A slight modification of the above argument shows (cf. Theorem 3.3) that $W\left(X^{*}, \mathcal{Q}^{\bullet}\right)$ is defined, and $F \mathcal{R} \mathcal{M}(X)$ is $(2,1,1)$-embeddable in $W\left(X^{*}, \mathcal{Q}^{\bullet}\right)$. Furthermore, this embedding provides (cf. Corollary 3.4) a model of the free restriction monoid on $X$, denoted by $F_{W} \mathcal{R} \mathcal{M}(X)$, which is a $(2,1,1)$-subsemigroup in $W\left(X^{*}, \mathcal{Q}^{\bullet}\right)$.

(2) Although $P\left(F \mathcal{G}(X), \mathcal{X}^{\bullet}, \mathcal{Y}^{\bullet}\right)$ is just $P(F \mathcal{G}(X), \mathcal{X}, \mathcal{Y})$ with an identity element adjoined and, similarly, $F \mathcal{R} \mathcal{M}(X)$ is just $F \mathcal{R S}(X)$ with an identity element adjoined, this is definitely not the case with $W\left(X^{*}, \mathcal{Q}^{\bullet}\right)$ and $W\left(X^{*}, \mathcal{Q}\right)$. Namely, $W\left(X^{*}, \mathcal{Q}^{\bullet}\right)$ is not a monoid (cf. Result 2.1(4)).

\section{Proper covers and $W$-Products}

In this section we show that each restriction semigroup has a proper cover (actually, a proper ample cover) which is embeddable in a $W$-product of a semilattice by a monoid. This is obtained by proving that each $(2,1,1)$ factor semigroup of $F_{W} \mathcal{R S}(X)$ over a $(2,1,1)$-congruence contained in $\sigma$ is $(2,1,1)$-embeddable in a $W$-product of a semilattice by a monoid (cf. Result 2.6 and Corollary 3.4).

Let $\rho$ be a $(2,1,1)$-congruence on $F_{W} \mathcal{R S}(X)$ with $\rho \subseteq \sigma$. Then, for any $\left(t, A^{t}\right),\left(u, B^{u}\right) \in F_{W} \mathcal{R} \mathcal{S}(X)$,

$$
\left(t, A^{t}\right) \rho\left(u, B^{u}\right) \text { implies } t=u \text {. }
$$

The restriction of $\rho$ to the semilattice of projections $P_{F_{W} \mathcal{R S}(X)}=\{(1, A)$ : $A \in \mathcal{Y}\}$ is a congruence. By Result 2.4(3), the semilattice $P_{F_{W} \mathcal{R S}(X)}$ is isomorphic to $\mathcal{Y}$, therefore the relation

$$
\kappa_{\rho}=\{(A, B) \in \mathcal{Y} \times \mathcal{Y}:(1, A) \rho(1, B)\}
$$

is a congruence on $\mathcal{Y}$. It relates to $\rho$ as follows.

Lemma 4.1. For any $\left(t, A^{t}\right),\left(u, B^{u}\right) \in F_{W} \mathcal{R S}(X)$, the following conditions are equivalent: 
(1) $\left(t, A^{t}\right) \rho\left(u, B^{u}\right)$,

(2) $t=u$ and $A \kappa_{\rho} B$,

(3) $t=u$ and $A^{t} \kappa_{\rho} B^{t}$.

Proof. Since $\rho \subseteq \sigma$, property (1) implies $t=u$. Moreover, $\rho$ is a $(2,1,1)$ congruence, therefore (1) implies $(1, A)=\left(t, A^{t}\right)^{+} \rho\left(t, B^{t}\right)^{+}=(1, B)$ and $\left(1, A^{t}\right)=\left(t, A^{t}\right)^{*} \rho\left(t, B^{t}\right)^{*}=\left(1, B^{t}\right)$. Thus (1) implies (2) and (3).

Before considering the reverse implications, notice that $A \in \mathcal{Y}$ and $t \in A$, and so $[t]^{\downarrow} \subseteq A$ is implied. Since $[t]^{\downarrow} \in \mathcal{Y}$, we have $\left(t,[t]^{\downarrow}\right) \in F_{W} \mathcal{R} \mathcal{S}(X)$, and so

$$
(1, A)\left(t,[t]^{\downarrow^{t}}\right)=\left(t, A^{t}\right)=\left(t,[t]^{\downarrow^{t}}\right)\left(1, A^{t}\right) .
$$

Consequently, either we have $A \kappa_{\rho} B$ or $A^{t} \kappa_{\rho} B^{t}$, that is, either we have $(1, A) \rho(1, B)$ or $\left(1, A^{t}\right) \rho\left(1, B^{t}\right)$, we obtain that $\left(t, A^{t}\right) \rho\left(t, B^{t}\right)=\left(u, B^{u}\right)$. This shows that each of (2) and (3) implies (1).

The $(2,1,1)$-congruences of $F_{W} \mathcal{R S}(X)$ contained in $\sigma$ can be characterized in the following manner.

Proposition 4.2. Let $\kappa$ be a congruence on $\mathcal{Y}$ such that the following condition is fulfilled:

(P) for every $A, B \in \mathcal{Y}$ and $t \in X^{*} \cap A \cap B$, we have $A \kappa B$ if and only if $A^{t} \kappa B^{t}$.

Then the relation $\rho$ defined by the rule

$$
\left(t, A^{t}\right) \rho\left(u, B^{u}\right) \text { if and only if } t=u \text { and } A \kappa B
$$

is a $(2,1,1)$-congruence on $F_{W} \mathcal{R S}(X)$ contained in $\sigma$. Conversely, each $(2,1,1)$-congruence on $F_{W} \mathcal{R S}(X)$ contained in $\sigma$ can be obtained in this way.

Proof. The last statement immediately follows from Lemma 4.1 if we recall that, for some $A \in \mathcal{Y}$ and $t \in X^{*}$, we have $\left(t, A^{t}\right) \in F_{W} \mathcal{R S}(X)$ if and only if $t \in A$.

Assume that $\kappa$ is a congruence on $\mathcal{Y}$ such that condition $(\mathrm{P})$ is satisfied, and consider the relation $\rho$ on $F_{W} \mathcal{R S}(X)$ defined by $\kappa$ as in the statement. Obviously, $\rho \subseteq \sigma$. We check that $\rho$ is a $(2,1,1)$-congruence. Let $\left(t, A^{t}\right) \rho\left(u, B^{u}\right)$ in $F_{W} \mathcal{R S}(X)$. Then we have $t=u$ and $A \kappa B$, and the latter relation implies $A^{t} \kappa B^{t}$ by $(\mathrm{P})$. Hence $\left(t, A^{t}\right)^{+}=(1, A) \rho(1, B)=\left(t, B^{t}\right)^{+}$ and $\left(t, A^{t}\right)^{*}=\left(1, A^{t}\right) \rho\left(1, B^{t}\right)=\left(t, B^{t}\right)^{*}$ are straightforward, and so $\rho$ is compatible with both unary operations. Moreover, for any $\left(v, C^{v}\right) \in$ $F_{W} \mathcal{R S}(X)$, we have

$$
\left(v, C^{v}\right)\left(t, A^{t}\right)=\left(v t,\left({ }^{v}\left(C^{v} \cup A\right)\right)^{v t}\right), \quad\left(v, C^{v}\right)\left(t, B^{t}\right)=\left(v t,\left({ }^{v}\left(C^{v} \cup B\right)\right)^{v t}\right)
$$

and

$$
\left(t, A^{t}\right)\left(v, C^{v}\right)=\left(t v,\left({ }^{t}\left(A^{t} \cup C\right)\right)^{t v}\right), \quad\left(t, B^{t}\right)\left(v, C^{v}\right)=\left(t v,\left({ }^{t}\left(B^{t} \cup C\right)\right)^{t v}\right) .
$$

Here $A, B, C^{v}, A^{t}, B^{t}, C \in \mathcal{Y}$, and since $\kappa$ is a congruence on $\mathcal{Y}$, we deduce that

$$
C^{v} \cup A \kappa C^{v} \cup B \text { and } A^{t} \cup C \kappa B^{t} \cup C .
$$

The sets in the first pair contain $1, v^{-1}$ and those in the second one contain $1, t^{-1}$ because $1 \in A, B, C$. Thus ${ }^{v}\left(C^{v} \cup A\right),{ }^{v}\left(C^{v} \cup B\right),{ }^{t}\left(A^{t} \cup C\right),{ }^{t}\left(B^{t} \cup C\right) \in$ 
$\mathcal{Y}$, we have $v \in{ }^{v}\left(C^{v} \cup A\right) \cap{ }^{v}\left(C^{v} \cup B\right), t \in{ }^{t}\left(A^{t} \cup C\right) \cap{ }^{t}\left(B^{t} \cup C\right)$, and the relations in (4.1) can be written in the form

$$
\left({ }^{v}\left(C^{v} \cup A\right)\right)^{v} \kappa\left({ }^{v}\left(C^{v} \cup B\right)\right)^{v} \text { and } \quad\left({ }^{t}\left(A^{t} \cup C\right)\right)^{t} \kappa\left({ }^{t}\left(B^{t} \cup C\right)\right)^{t},
$$

respectively. By applying property $(\mathrm{P})$ in the reverse direction, hence we obtain that ${ }^{v}\left(C^{v} \cup A\right) \kappa^{v}\left(C^{v} \cup B\right)$ and ${ }^{t}\left(A^{t} \cup C\right) \kappa^{t}\left(B^{t} \cup C\right)$. This implies $\left(v, C^{v}\right)\left(t, A^{t}\right) \rho\left(v, C^{v}\right)\left(t, B^{t}\right)$ and $\left(t, A^{t}\right)\left(v, C^{v}\right) \rho\left(t, B^{t}\right)\left(v, C^{v}\right)$ by definition. Thus $\rho$ is compatible also with the multiplication.

Let $\kappa$ be any congruence on $\mathcal{Y}$ such that condition $(\mathrm{P})$ is satisfied. We extend $\kappa$ from $\mathcal{Y}$ to $\mathcal{Q}$. First we define a relation $\nu_{0}$ as follows: for any $P, Q \in \mathcal{Q} \subseteq \mathcal{X}$, let $P \nu_{0} Q$ if there exists $t \in X^{*}$ such that ${ }^{t} P,{ }^{t} Q \in \mathcal{Y}$ and ${ }^{t} P \kappa^{t} Q$ in $\mathcal{Y}$. It is obvious by definition that the relation $\nu_{0}$ is reflexive and symmetric. Now we show further properties of $\nu_{0}$.

Lemma 4.3. The relation $\nu_{0}$ is left and right compatible on $(\mathcal{Q} ; \vee)$.

Proof. Since the operation $\vee$ is commutative, it suffices to verify right compatibility. Let $P, Q, R \in \mathcal{Q}$ such that $P \nu_{0} Q$, that is, there exists $t \in X^{*}$ such that ${ }^{t} P,{ }^{t} Q \in \mathcal{Y}$ with ${ }^{t} P \kappa{ }^{t} Q$. We intend to show that $P \vee R \nu_{0} Q \vee R$. By definition, we have $u \in X^{*}$ with $u^{-1} \in R$. By Result 2.2(1), we see that $P \vee R={ }^{-1}\left({ }^{t} P \cup\left[t u^{-1}\right]^{\downarrow} \cup{ }^{t} R\right)$ and $Q \vee R={ }^{-1}\left({ }^{t} Q \cup\left[t u^{-1}\right]^{\downarrow} \cup{ }^{t} R\right)$. Hence ${ }^{t}(P \vee R)={ }^{t} P \cup\left[t u^{-1}\right]^{\downarrow} \cup{ }^{t} R$ and ${ }^{t}(Q \vee R)={ }^{t} Q \cup\left[t u^{-1}\right]^{\downarrow} \cup{ }^{t} R$, where ${ }^{t} P \kappa^{t} Q$ in $\mathcal{Y}$ and $\left[t u^{-1}\right]^{\downarrow} \cup{ }^{t} R \in \mathcal{Y}$. This implies ${ }^{t}(P \vee R) \kappa^{t}(Q \vee R)$ since $\kappa$ is a congruence on $\mathcal{Y}$, and so $P \vee R \nu_{0} Q \vee R$ follows by the definition of $\nu_{0}$.

Lemma 4.4. For every $P, Q \in \mathcal{Q}$ and $v \in X^{*}$, we have $P \nu_{0} Q$ if and only if $P^{v} \nu_{0} Q^{v}$.

Proof. Let $v \in X^{*}$ and $P, Q \in \mathcal{Q}$. Assume first that $P \nu_{0} Q$, that is, there exists $u \in X^{*}$ such that ${ }^{u} P,{ }^{u} Q \in \mathcal{Y}$ with ${ }^{u} P \kappa^{u} Q$. Then we obviously have ${ }^{u v}\left(P^{v}\right)={ }^{u}\left({ }^{v}\left(P^{v}\right)\right)={ }^{u} P \kappa^{u} Q={ }^{u}\left({ }^{v}\left(Q^{v}\right)\right)={ }^{u v}\left(Q^{v}\right)$, which implies $P^{v} \nu_{0} Q^{v}$.

Conversely, suppose that $P^{v} \nu_{0} Q^{v}$. Then there exists $u \in X^{*}$ such that ${ }^{u}\left(P^{v}\right),{ }^{u}\left(Q^{v}\right) \in \mathcal{Y}$ with ${ }^{u}\left(P^{v}\right) \kappa^{u}\left(Q^{v}\right)$. In particular, this implies that $1 \in$ ${ }^{u}\left(P^{v}\right) \cap{ }^{u}\left(Q^{v}\right)$ whence $v u^{-1} \in P \cap Q$ follows. Now we distinguish two cases according to whether $u=z v$ for some $z \in X^{*}$ or not.

Assume that $u=z v$ for some $z \in X^{*}$. Then ${ }^{z} P={ }^{u v^{-1}} P={ }^{u}\left(P^{v}\right) \in \mathcal{Y}$ and, similarly, ${ }^{z} Q={ }^{u}\left(Q^{v}\right) \in \mathcal{Y}$. Therefore, by assumption, we have ${ }^{z} P \kappa^{z} Q$, and so $P \nu_{0} Q$ is implied by definition.

Turning to the opposite case, assume that $u \neq z v$ for any $z \in X^{*}$. This means that $u=u_{1} r$ and $v=v_{1} r$ for some $r, u_{1} \in X^{*}$ and $v_{1} \in X^{*} \backslash\{1\}$ where $\operatorname{red}\left(u_{1} v_{1}^{-1}\right)=u_{1} v_{1}^{-1}$. Since $P, Q \in \mathcal{Q}$, we have $s, t \in X^{*}$ such that $s^{-1} \in P$ and $t^{-1} \in Q$. Therefore we deduce that

$$
u(s v)^{-1} \in{ }^{u}\left(P^{v}\right) \text { and } u(t v)^{-1} \in{ }^{u}\left(Q^{v}\right) .
$$

Since ${ }^{u}\left(P^{v}\right) \in \mathcal{Y}$, the first relation implies that $\left[u(s v)^{-1}\right]^{\downarrow} \subseteq{ }^{u}\left(P^{v}\right)$. Moreover, we have $\operatorname{red}\left(u(s v)^{-1}\right)=\operatorname{red}\left(u v^{-1} s^{-1}\right)=\operatorname{red}\left(u_{1} v_{1}^{-1} s^{-1}\right)=u_{1} v_{1}^{-1} s^{-1}$ by the choice of $u_{1}$ and $v_{1}$. This implies that $u_{1}, u_{1} v_{1}^{-1} \in{ }^{u}\left(P^{v}\right)$, and a similar argument shows that we also have $u_{1}, u_{1} v_{1}^{-1} \in{ }^{u}\left(Q^{v}\right)$. Therefore we can apply property $(\mathrm{P})$ for the $\kappa$-related elements ${ }^{u}\left(P^{v}\right),{ }^{u}\left(Q^{v}\right) \in \mathcal{Y}$ and for the word 
$u_{1}$, and we obtain that $P^{v_{1}}={ }^{r}\left(P^{v}\right)=\left({ }^{u}\left(P^{v}\right)\right)^{u_{1}} \kappa\left({ }^{u}\left(Q^{v}\right)\right)^{u_{1}}={ }^{r}\left(Q^{v}\right)=Q^{v_{1}}$. Recalling that $u_{1} v_{1}^{-1} \in{ }^{u}\left(P^{v}\right)$, we see that $v_{1}^{-1} \in\left({ }^{u}\left(P^{v}\right)\right)^{u_{1}}=P^{v_{1}}$ and, similarly, that $v_{1}^{-1} \in Q^{v_{1}}$. Hence $1 \in P, Q$ follows. On the other hand, we have seen that $P^{v_{1}}, Q^{v_{1}} \in \mathcal{Y}$, and so $v_{1} \in P, Q$ is also implied. Thus $P, Q \in \mathcal{Y}$ such that $P^{v_{1}} \kappa Q^{v_{1}}$ in $\mathcal{Y}$. Therefore we deduce by property $(\mathrm{P})$ that $P \kappa Q$ which implies $P \nu_{0} Q$.

Lemma 4.5. If $A, B \in \mathcal{Y}$ such that $A \nu_{0} B$ then $A \kappa B$.

Proof. Let $A, B \in \mathcal{Y}$ such that $A \nu_{0} B$. By definition, there exists $u \in X^{*}$ such that ${ }^{u} A,{ }^{u} B \in \mathcal{Y}$ and ${ }^{u} A \kappa{ }^{u} B$. Here $A, B \in \mathcal{Y}$ implies $u \in{ }^{u} A,{ }^{u} B$. Therefore property $(\mathrm{P})$ implies $A=\left({ }^{u} A\right)^{u} \kappa\left({ }^{u} B\right)^{u}=B$.

Now let us consider the equivalence relation on $\mathcal{Q}$ generated by $\nu_{0}$, and denote it by $\nu$. Since $\nu_{0}$ is reflexive and symmetric, $\nu$ is the transitive closure of $\nu_{0}$. Thus, for every $P, Q \in \mathcal{Q}$, we have $P \nu Q$ if and only if there exists a sequence $R_{i}(i=0,1, \ldots, k)$ of elements of $\mathcal{Q}$ such that

$$
P=R_{0} \nu_{0} R_{1} \nu_{0} R_{2} \nu_{0} \ldots \nu_{0} R_{k}=Q .
$$

The following lemma extends the properties of $\nu_{0}$ verified so far to $\nu$.

Lemma 4.6. The relation $\nu$ is a congruence on the semilattice $\mathcal{Q}=(\mathcal{Q} ; \vee)$ such that, for every $P, Q \in \mathcal{Q}$ and $v \in X^{*}$, we have $P \nu Q$ if and only if $P^{v} \nu Q^{v}$.

Proof. Lemma 4.3 immediately implies that $\nu$ is left and right compatible on $(\mathcal{Q} ; \vee)$, and since $\nu$ is an equivalence relation, we obtain that it is a congruence on the semilattice $\mathcal{Q}$. It is also straightforward by the 'only if' part of Lemma 4.4 that if $P \nu Q$ in $\mathcal{Q}$ and $v \in X^{*}$ then $P^{v} \nu Q^{v}$ follows.

Now suppose that $P, Q \in \mathcal{Q}$ and $v \in X^{*}$ such that $P^{v} \nu Q^{v}$. Then there exists a sequence $R_{i}(i=0,1, \ldots, k)$ of elements of $\mathcal{Q}$ such that

$$
P^{v}=R_{0} \nu_{0} R_{1} \nu_{0} R_{2} \nu_{0} \ldots \nu_{0} R_{k}=Q^{v} .
$$

Applying Lemma 4.3, we infer from these relations that

$P^{v}=P^{v} \vee R_{0} \nu_{0} P^{v} \vee R_{1} \nu_{0} P^{v} \vee R_{2} \nu_{0} \ldots \nu_{0} P^{v} \vee R_{k}=P^{v} \vee Q^{v}=(P \vee Q)^{v}$.

Since $\mathcal{Q}^{v}$ is a dual order ideal by Lemma 3.2, there exists $S_{i} \in \mathcal{Q}$ with $S_{i}^{v}=P^{v} \vee R_{i}$ for each $i(i=0,1, \ldots, k)$. Thus we have

$$
P^{v}=S_{0}^{v} \nu_{0} S_{1}^{v} \nu_{0} S_{2}^{v} \nu_{0} \ldots \nu_{0} S_{k}^{v}=(P \vee Q)^{v},
$$

whence, by Lemma 4.4, we deduce that

$$
P=S_{0} \nu_{0} S_{1} \nu_{0} S_{2} \nu_{0} \ldots \nu_{0} S_{k}=P \vee Q
$$

This shows that $P \nu P \vee Q$. By symmetry, we also have $Q \nu P \vee Q$, and so the relation $P \nu Q$ is verified.

Another property of $\nu$ which is crucial for the main result of this section is that its restirction to $\mathcal{Y}$ is $\kappa$.

Lemma 4.7. For any $A, B \in \mathcal{Y}(\subseteq \mathcal{Q})$, the relation $A \nu B$ implies $A \kappa B$. 
Proof. Assume that $A, B \in \mathcal{Y}$ such that $A \nu B$. Then there exists a sequence $R_{i}(i=0,1, \ldots, k)$ of elements of $\mathcal{Q}$ such that

$$
A=R_{0} \nu_{0} R_{1} \nu_{0} R_{2} \nu_{0} \ldots \nu_{0} R_{k}=B .
$$

By Lemma 4.3, this implies

$$
A=A \vee R_{0} \nu_{0} A \vee R_{1} \nu_{0} A \vee R_{2} \nu_{0} \ldots \nu_{0} A \vee R_{k}=A \vee B .
$$

Here $A \in \mathcal{Y}$, that is, $1 \in A$, whence we see that $A \vee R_{i} \in \mathcal{Y}$ for every $i(i=0,1, \ldots, k)$. Applying Lemma 4.5 for $(4.3)$, we obtain that

$$
A=A \vee R_{0} \kappa A \vee R_{1} \kappa A \vee R_{2} \kappa \ldots \kappa A \vee R_{k}=A \vee B,
$$

whence $A \kappa A \vee B$ follows. By symmetry, we also have $B \kappa A \vee B$ which implies $A \kappa B$.

By Lemma 4.6, we can consider the factor semilattice $\mathcal{Q} / \nu$, and denote the partial order on it by $\geq$; that is, we write $P \nu \geq Q \nu$ if $P \nu \vee Q \nu=P \nu$. Also by Lemma 4.6, the right action of $X^{*}$ on $\mathcal{Q}$ induces a right action of $X^{*}$ on $\mathcal{Q} / \nu$ defined by the rule $(Q \nu)^{v}=\left(Q^{v}\right) \nu\left(Q \in \mathcal{Q}, v \in X^{*}\right)$. Moreover, the endomorphism of $\mathcal{Q} / \nu$ corresponding to $v$ is injective for every $v \in X^{*}$. In order to be able to define $W\left(X^{*}, \mathcal{Q} / \nu\right)$, we need the following property.

Lemma 4.8. For any $t \in X^{*}$, the set $(\mathcal{Q} / \nu)^{t}$ forms a dual order ideal in $\mathcal{Q} / \nu$.

Proof. Let $P, Q \in \mathcal{Q}$ and $t \in X^{*}$ such that $P \nu \geq(Q \nu)^{t}$ in $\mathcal{Q} / \nu$. We intend to verify that $P \nu \in(\mathcal{Q} / \nu)^{t}$. The inequality assumed is equivalent to the equality $P \nu \vee(Q \nu)^{t}=P \nu$, and so, by definition, to the relation $P \vee Q^{t} \nu P$. Since $\mathcal{Q}^{t}$ is a dual order ideal in $\mathcal{Q}$ by Lemma 3.2, we obtain that $P \vee Q^{t}=S^{t}$ for some $S \in \mathcal{Q}$. Hence $P \nu=\left(P \vee Q^{t}\right) \nu=\left(S^{t}\right) \nu=(S \nu)^{t} \in(\mathcal{Q} / \nu)^{t}$.

Thus we have established that $W\left(X^{*}, \mathcal{Q} / \nu\right)$ is defined since all the conditions required for this definition are satisfied. Furthermore, the mapping $W\left(X^{*}, \mathcal{Q}\right) \rightarrow W\left(X^{*}, \mathcal{Q} / \nu\right),\left(t, Q^{t}\right) \mapsto\left(t,(Q \nu)^{t}\right)$ is clearly a $(2,1,1)$ morphism. Restricting it to $F_{W} \mathcal{R S}(X)$, we obtain the $(2,1,1)$-morphism $\phi: F_{W} \mathcal{R S}(X) \rightarrow W\left(X^{*}, \mathcal{Q} / \nu\right)$ defined by $\left(t, A^{t}\right) \phi=\left(t,(A \nu)^{t}\right)$. Lemma 4.7 implies that, for any $\left(t, A^{t}\right),\left(u, B^{u}\right) \in F_{W} \mathcal{R S}(X)$, we have $\left(t, A^{t}\right) \phi=$ $\left(u, B^{u}\right) \phi$ if and only if $t=u$ and $A \kappa B$. For, if $\left(t, A^{t}\right) \phi=\left(u, B^{u}\right) \phi$ then $t=u$ and $(A \nu)^{t}=(B \nu)^{u}$ follows by definition. Hence $A \nu=B \nu$ is deduced, since $t=u$ and the endomorphism of $\mathcal{Q} / \nu$ corresponding to $t$ is injective. Here $A, B \in \mathcal{Y}$, therefore Lemma 4.7 ensures $A \kappa B$. Thus the congruence induced by $\phi$ is the $(2,1,1)$-congruence $\rho$ corresponding to $\kappa$, see Proposition 4.2. This implies that the restriction semigroup $F_{W} \mathcal{R} \mathcal{S}(X) / \rho$ is $(2,1,1)$-embeddable in $W\left(X^{*}, \mathcal{Q} / \nu\right)$. By applying Proposition 4.2 , we can summarize the result proved so far as follows.

Proposition 4.9. Suppose that $\rho$ is a $(2,1,1)$-congruence on $F_{W} \mathcal{R S}(X)$ contained in $\sigma$. Denote by $\kappa$ the congruence on $\mathcal{Y}$ corresponding to $\rho$ by Proposition 4.2, and consider the congruence $\nu$ on the semilattice $\mathcal{Q}$ generated by the relation

$$
\left\{(P, Q) \in \mathcal{Q} \times \mathcal{Q}:{ }^{t} P,{ }^{t} Q \in \mathcal{Y} \text { with }{ }^{t} P \kappa{ }^{t} Q \text { for some } t \in X^{*}\right\} .
$$


Then the right action of $X^{*}$ on $\mathcal{Q}$ induces a right action of $X^{*}$ on the factor semilattice $\mathcal{Q} / \nu$ by putting $(Q \nu)^{v}=\left(Q^{v}\right) \nu$ for every $Q \in \mathcal{Q}, v \in X^{*}$, such that $W\left(X^{*}, \mathcal{Q} / \nu\right)$ is defined, and the mapping

$$
F_{W} \mathcal{R S}(X) / \rho \rightarrow W\left(X^{*}, \mathcal{Q} / \nu\right),\left(t, A^{t}\right) \rho \mapsto\left(t,(A \nu)^{t}\right)
$$

is a $(2,1,1)$-embedding.

This is, actually, the main result of this section which can be formulated, in a less technical way, as follows.

Theorem 4.10. Each $(2,1,1)$-factor semigroup of a free restriction semigroup over a $(2,1,1)$-congruence contained in the least unary trivial $(2,1,1)$ congruence (equivalently, in the least cancellative congruence) is $(2,1,1)$ embeddable in a $W$-product of a semilattice by a monoid.

By Result 2.6, we deduce the statement we aimed to prove in the paper.

Theorem 4.11. Each restriction semigroup has a proper (ample) cover which is $(2,1,1)$-embeddable into a $W$-product of a semilattice by a monoid.

Remark 4.12. The analogue of Proposition 4.9 where $F_{W} \mathcal{R S}(X)$ is replaced by $F_{W} \mathcal{R} \mathcal{M}(X)$ and $\mathcal{Q}$ by $\mathcal{Q}^{\bullet}$ also holds.

Note that the main result of [6] proves that each restriction semigroup is $(2,1,1)$-embeddable into a so-called almost left factorizable restriction semigroup. A restriction semigroup is almost left factorizable if and only if it has a proper cover isomorphic to a $W$-product of a semilattice by a monoid. Thus the main result of [6] is, in some sense, dual to Theorem 4.11.

\section{REFERENCES}

[1] J. Fountain and G. M. S. Gomes, Proper left type- $A$ monoids revisited, Glasgow Math. J. 35 (1993), 293-306.

[2] J. Fountain, G. M. S. Gomes and V. Gould, The free ample monoid, Internat. J. Algebra Comput. 19 (2009), 527-554.

[3] G. M. S. Gomes and M. B. Szendrei, Almost factorizable weakly ample semigroups, Comm. Algebra 35 (2007), 3503-3523.

[4] V. Gould, Notes on restriction semigroups and related structures; formerly (Weakly) left $E$-ample semigroups, http://www-users.york.ac.uk / varg1/gpubs.htm

[5] M. V. Lawson, Inverse semigroups: The Theory of Partial Symmetries, World Scientific, 1998.

[6] M. B. Szendrei, Embedding into almost left factorizable restriction semigroups, Comm. Algebra, to appear.

Bolyai Institute, University of Szeged, Aradi vértanúk tere 1, Szeged, HunGARY, H-6720

E-mail address: m.szendrei@math.u-szeged.hu 